\title{
Régimen laboral de las empresas públicas ecuatorianas
}

\author{
Ecuador's legal framework on labor for \\ Government-run companies
}

\section{Lizzeth Karina Villavicencio Logroño}

Investigadora jurídica independiente

Ciudad: Quito

Pais: Ecuador

Artículo original (investigación)

RFJ, No. 7, 2020, pp. 366-404, ISSN 2588-0837

RESUMEN: Las empresas públicas son una herramienta fundamental para el cumplimiento de los objetivos del Estado. Estas empresas de Estado, como también se las conoce, han sido dotadas de facultades conforme la utilidad que han ido adquiriendo. Debido a su importancia, se ha previsto un régimen jurídico particular que les permita asimilar su funcionamiento a las empresas privadas para que puedan competir en igualdad de condiciones y producir rentabilidad social.

En el mismo sentido, se ha contemplado que su régimen laboral diste de aquel que impera en el resto del sector púbico. A través de una metodología explicativa, el presente artículo acomete develar el régimen laboral que el legislador ecuatoriano ha previsto para los servidores públicos que trabajan en estas entidades. Desde una perspectiva analítica, se pretende concluir que se trata de un régimen mixto que no concuerda con lo establecido por la doctrina y que requiere ser unificado para evitar interpretaciones arbitrarias.

PALABRAS CLAVE: empresas públicas, servidores públicos, sector público, administración pública

ABSTRACT: Government-Run companies are a fundamental tool for achieving the State's objectives. These State-Owned 
companies, as they are also known, have been empowered according to the utility they have been acquiring. Due to their importance, a particular legal framework has been established, allowing them to assimilate their operation to private companies so that they can compete on equal terms and produce social profitability.

In the same matter, it has been suggested that their labor framework has to be different from those prevailing in the rest of the public sector. Through an analytical methodology, this article aims to unveil the labor legal framework that the Ecuadorian legislation has instituted for public servants who work in these entities. From an analytical perspective, it intends to conclude that a mixed legal framework does not agree with what is established by the legal doctrine and that it needs to be unified to avoid arbitrary interpretations.

KEYWORDS: Government-Run companies / State-owned enterprises, public servants, public sector, public administration.

\section{INTRODUCCIÓN}

El servicio público ha sido una arista de trascendental importancia para los Estados a lo largo de la historia ya que los servidores públicos son quienes han hecho posible el cumplimiento de los objetivos previstos por los gobernantes y legisladores. A pesar de que han existido diversas opiniones y se ha generado un debate continuo sobre el desarrollo de sus funciones y la trascendencia de su trabajo, es claro que son un pilar fundamental para la administración pública.

Debido al alcance de sus atribuciones, la naturaleza de la relación entre los servidores y la administración pública ha sido ampliamente analizada por la doctrina. Por ende, se ha establecido una clasificación macro para poder determinar de mejor manera las particularidades que revisten a los servidores públicos. Dicha clasificación no solo se ha basado en la funcionalidad o utilidad de cada tipo de servidor público, sino también ha tomado en consideración el tipo de entidades en las que prestan sus servicios. 
En especial, la naturaleza de la entidad a la que pertenecen ha cobrado importancia cuando la misma ha sido dotada de un régimen particular. Este es el caso de las empresas públicas o empresas comerciales e industriales -empresas de Estado-, como se las conoce en la doctrina, a las cuales los Estados han ido atribuyendo competencias según sus necesidades. A pesar de que las mismas no han podido ser definidas de manera unánime pues "desarrollan una actividad comercial o industrial con un régimen jurídico entremezclado que combina el Derecho Público con el Derecho Privado" (Cassagne, 1998, p.340), cada legislación las ha normado en correspondencia con su finalidad última.

Consecuentemente, el legislador ecuatoriano las ha convertido en una herramienta de suma trascendencia y las ha dotado de elementos únicos. Esta circunstancia se ha hecho especialmente notoria en el régimen laboral imperante en las mismas, pues a la regulación de su talento humano la identifica un régimen jurídico mixto, mismo que es esencial para que el Estado pueda cumplir con su rol de empresario y produzca rentabilidad social. Empero, al haber previsto un régimen divergente al resto del sector público, en varios de sus actos y contratos se han encontrado particularidades no previstas (Villegas, 2013).

Este estudio procura esclarecer el régimen laboral impuesto por el ordenamiento jurídico ecuatoriano a los trabajadores ${ }^{1}$ que prestan sus servicios en las empresas públicas. A través de un método descriptivo y analítico pretende determinar en un primer momento la definición, características y clasificación que tanto la doctrina como la legislación han previsto para la generalidad de servidores públicos.

Como segundo punto, pretende poner en contexto a las empresas públicas y dar a conocer el régimen laboral que ha sido sugerido por la doctrina debido a la naturaleza mixta que impera en sus actos y contratos. En el mismo sentido, analiza el origen de la regulación laboral prevista por la norma ecuatoriana en la cual convergen normas de Derecho Público y de

1 Como servidores que poseen relación laboral con las empresas públicas ecuatorianas se debe entender a los servidores de libre nombramiento y remoción, servidores de carrera y obreros. 
Derecho Privado. Para finalizar, concluye con la interpretación de los cuerpos legales de aplicación concurrente en la regulación de los servidores de carrera, con el fin de dar solución a las interrogantes que este régimen poco concordante ha generado $\mathrm{y}$, de esta manera, esclarecer el régimen laboral mixto que ha caracterizado a los trabajadores que pertenecen a las empresas públicas ecuatorianas.

\section{SERVIDORES PÚBLICOS}

La naturaleza, funciones y principios que rigen a los servidores públicos han sido objeto de diversas consideraciones. Sin duda el papel que desempeñan en miras al correcto desenvolvimiento de la administración pública ha generado criterios contrapuestos; empero, indiscutiblemente su funcionalidad los ha vuelto indispensables para el desarrollo de los objetivos de cada Estado. Por ese motivo, no deja de extrañar que sus particularidades no hayan sido estudiadas a profundidad y consecuentemente no se haya dado la importancia debida a regular de manera adecuada los actos y contratos de los que son partícipes.

La falta de análisis oportuno ha caracterizado a la generalidad de componentes que revisten al servicio público y, por ende, ello ha marcado también al régimen laboral que los vincula con la administración pública. Este actuar errático ha sido causa de interpretaciones inconsistentes sobre su naturaleza y regulación, mismas que atañen a este estudio. Para iniciar con el análisis de aquellas inconsistencias es necesario tener presente que, si bien es cierto, el sector público dista en muchas características del sector privado, no es menos cierto que el Derecho Laboral inició como un Derecho protector de todo vínculo laboral por lo cual, sería cuestionable que los principios y garantías sean discordantes por el simple hecho de que un trabajador preste sus servicios en uno u otro sector (González, 2017).

Previo a dar una noción sobre la naturaleza y principios que rigen a las relaciones laborales en el sector público y su diferencia con aquellas que se producen en el sector privado, es menester poner en contexto al servicio público. Al respecto, 
es importante mencionar que a pesar de que no se ha dado el tiempo y el espacio que se debería al estudio de las características que envuelven a los servidores públicos, la doctrina ha sido enfática en señalar su conceptualización y clasificación pues, dichas aristas son trascendentales para abordar su regulación en el ámbito laboral.

\subsection{Nociones generales sobre el servicio público}

En primer lugar, es oportuno partir de la funcionalidad que, a través de la ley especial, se le ha otorgado al servicio público en el ordenamiento jurídico ecuatoriano. La Ley Orgánica de Servicio Público -LOSEP- entre sus primeros artículos ha determinado:

Objetivo. - El servicio público y la carrera administrativa tienen por objetivo propender al desarrollo profesional, técnico y personal de las y los servidores públicos, para lograr el permanente mejoramiento, eficiencia, eficacia, calidad, productividad del Estado y de sus instituciones, mediante la conformación, el funcionamiento y desarrollo de un sistema de gestión del talento humano sustentado en la igualdad de derechos, oportunidades y la no discriminación. (LOSEP, 2010, art. 2)

Como se desprende de la norma, es lógico concluir que el servicio público está orientado al mejoramiento continuo profesional y de toda índole de los servidores públicos. Este anhelo no es sorpresivo pues, los servidores públicos son aquellos que prestan sus servicios con miras a la realización de los objetivos de la administración pública. No obstante, lo que podría llegar a sorprender es que una ley orientada a regular al talento humano haga hincapié en principios exclusivos como aquellos mencionados en el primer artículo que establece:

Principios. - La presente Ley se sustenta en los principios de: calidad, calidez, competitividad, continuidad, descentralización, desconcentración, eficacia, eficiencia, equidad, igualdad, jerarquía, lealtad, oportunidad, 
participación, racionalidad, responsabilidad, solidaridad, transparencia, unicidad y universalidad que promuevan la interculturalidad, igualdad y la no discriminación. (LOSEP, 2010, art. 1)

Al sector público le rigen y amparan principios orientados al correcto funcionamiento de la administración pública pues el cumplimiento de sus planes es de interés público y, por ende, todo lo que se relacione con ello - más aún si se trata de sus servidores - debe tomar como base el beneficio de los administrados. Por otra parte, ello no impide que los principios que rigen al interés particular de los trabajadores, que en el caso de la disciplina laboral se encuentran plasmados en su gran mayoría en el Código del Trabajo, les sean aplicables cuando un servidor público guarda relación laboral con la administración pública.

Entonces, claramente la LOSEP al ser una norma que rige al servicio público debe hacer hincapié en los principios que rigen a la administración pública y que están encaminados a la protección de los administrados. No obstante, ello no implica que aquellos principios reconocidos a través del Derecho del Trabajo hayan sido desconocidos para los servidores públicos pues, sería incorrecto concluir que la categoría de servidor público excluye a la de trabajador, cuando en realidad la contiene. Simplemente, ante las diversas consideraciones que se han tenido al respecto, es necesario aclarar que además de los principios que rigen en la disciplina de Derecho Administrativo, cuando el trabajador pertenece al sector público y por ende es un servidor público, también le son aplicables otros principios concernientes a sus funciones individuales (Krotoschin, 1977).

\subsection{Aproximación conceptual a la denominación servidor público}

El Derecho Laboral es un Derecho protector de todo trabajador, independientemente del lugar o sector al que pertenezca y en el que realice sus labores. Es por ello, que no hay que olvidar que los servidores públicos también pueden ostentar la calidad de trabajadores dependiendo de sus funciones y el tipo 
de vínculo que mantengan con la administración pública. Para el efecto, es conveniente partir de la definición que ha otorgado la Carta Magna ecuatoriana al concepto "servidor público" misma que, cabe adelantar, concuerda en varios elementos con aquella otorgada por la doctrina; sin embargo, varios de los preceptos infra constitucionales han desviado en cierta forma dicha conceptualización. En su articulado la Carta Constitucional menciona:

Serán servidoras o servidores públicos todas las personas que en cualquier forma o a cualquier título trabajen, presten servicios o ejerzan un cargo, función o dignidad dentro del sector público.

Los derechos de las servidoras y servidores públicos son irrenunciables. La ley definirá el organismo rector en materia de recursos humanos y remuneraciones para todo el sector público y regulará el ingreso, ascenso, promoción, incentivos, régimen disciplinario, estabilidad, sistema de remuneración y cesación de funciones de sus servidores.

Las obreras y obreros del sector público estarán sujetos al Código de Trabajo.

La remuneración de las servidoras y servidores públicos será justa y equitativa, con relación a sus funciones, y valorará la profesionalización, capacitación, responsabilidad y experiencia. (CRE, 2008, art. 229)

El articulo precedente no solo da una definición amplia sobre la denominación "servidor público", sino que realiza una breve explicación acerca de ciertos elementos importantes al respecto como son los principios y parte de la normativa aplicable a los mismos, lo cual da una noción sobre el régimen aplicable. La conceptualización expresada por la Constitución ha sido reiterada en la normativa especial aplicable a la regulación del Talento Humano en el sector público. La Ley Orgánica de Servicio Público -LOSEP-, en sus primeros artículos contiene la definición mencionada y al respecto indica que: "Servidoras 
y servidores públicos. - Serán servidoras o servidores públicos todas las personas que en cualquier forma o a cualquier título trabajen, presten servicios o ejerzan un cargo, función o dignidad dentro del sector público" (LOSEP, 2010, art. 4).

La definición adoptada por la normativa ecuatoriana concuerda con la definición otorgada por una gran parte de la doctrina, misma que en breves palabas ha establecido que:

En simple descripción o definición diremos que toda persona que proporcione un servicio o actividad remunerada en beneficio del Estado. Independientemente de la denominación que se le haya proporcionado al trabajador (servidor, funcionario o empleado público), este cumple un mismo fin, el de servir a nombre del Estado, a sus mandantes. (Valencia, 2015, p.10)

Tanto la norma como la doctrina han sido enfáticos en señalar que servidor público es una categoría que atañe a toda persona que, independientemente de la denominación que se le otorgue, presta sus servicios, trabaja o realiza cualquier actividad encaminada al beneficio de los fines de la administración pública. Entre líneas, la conceptualización realizada ha intentado cubrir los múltiples errores conceptuales que se han generado alrededor de esta denominación pues no solo en la legislación, sino que incluso en la doctrina, ha existido confusión en varios elementos. Por ejemplo, se ha generado una errónea conclusión en cuanto a la supuesta sinonimia existente entre el concepto de funcionario y servidor público o en la supuesta antonimia entre el concepto de trabajador y servidor público misma que, como es lógico, carece de sentido (Bielsa, 1980).

Las interrogantes descritas no son las únicas que han causado confusión alrededor del concepto servidor público, pues de las mismas han nacido ciertas actuaciones arbitrarias por el simple hecho de no conocer a profundidad las características que rigen al servicio público y al Derecho Laboral. Previo a desarrollar la interrogante que interesa a este estudio, es oportuno presentar un ejemplo de lo que ha conllevado el desconocimiento mencionado. 
En el año 2015, a través de una serie de enmiendas constitucionales, los legisladores dieron un giro a varios ámbitos que se encontraban previstos, entre ellos el laboral. A través de estas enmiendas constitucionales se produjeron cambios en los artículos 229 y 326 de la Constitución ecuatoriana mismos que hacen referencia al régimen laboral de los obreros en el sector público. Los legisladores intentaron justificar dicho cambio en base al principio de igualdad, aduciendo que el nuevo régimen lograría el tan anhelado trato igualitario de todos los servidores públicos y coincidiría con los preceptos sugeridos por la OIT (Sector público, con nuevas reglas para contratación de trabajadores, 2015).

Claramente en dicha motivación el legislador no tomó en cuenta que los principios de Derecho Laboral les son aplicables a los obreros a pesar de que son considerados servidores públicos. Esta errónea aplicación de la normativa causó que la Corte Constitucional determinara la inconstitucionalidad de estas enmiendas poco después, en el año 2018 (CC-SIN-018-18, 2018). Al respecto es importante recordar que gran parte la doctrina ha criticado estos cambios abruptos en la legislación en cuanto al régimen de los servidores públicos, en especial porque considera que la diferenciación o semejanza que se haga debe sobrepasar una concepción etimológica, pues el primer paso sería velar por la aplicación en igualdad de condiciones de los derechos laborales y posterior a ello unificar el régimen aplicable a los servidores públicos. (Trujillo, 2008).

\subsection{Clasificación doctrinaria de los servidores públicos}

Una vez analizada la conceptualización y radicada la importancia del servicio público en cuanto a su utilidad para el logro de los objetivos de todo Estado, es importante recordar que la normativa debe ir encaminada a cumplir con dicha finalidad. Por ese motivo, tanto la normativa como la doctrina han realizado una clasificación base y han determinado, conforme a la misma, las funciones y particularidades que deben revestir a los servidores públicos en sus cargos. En gran parte, esta clasificación ha sido basada en parámetros objetivos como la modalidad de vinculación con el sector público (Valencia, 2015). 
La gran mayoría de servidores públicos trabajan para el sector público en el ámbito de sus competencias y por ello, el grupo que interesa para este estudio son aquellos que mantienen una relación laboral con el Estado ${ }^{2}$. Los servidores públicos que se encuentran en el grupo mencionado, han sido catalogados por la doctrina como empleados oficiales con la finalidad de asimilar su denominación a los trabajadores del sector privado y así, disminuir en alguna forma la brecha existente entre unos y otros. Para establecer una clasificación adecuada se ha tomado en cuenta principalmente que: "El vínculo laboral con la administración pública, se manifiesta de maneras diferentes: una modalidad estatutaria, otra contractual y una tercera de carácter temporal que agrupa a quienes han sido denominados en la doctrina como auxiliares de la administración" (Dueñas, 2009, p. 64).

Consecuentemente, con base en la modalidad de vinculación, los empleados oficiales han sido divididos en dos grupos principales que son empleados públicos y trabajadores oficiales. El primer grupo se caracteriza porque sus servidores se vinculan con la administración pública a través de una modalidad estatutaria, legal o reglamentaria; en cambio, los servidores del segundo grupo pasan a formar parte de la administración pública mediante una modalidad contractual (Dueñas, 2009).

La modalidad estatutaria se refiere al proceso mediante el cual una persona pasa a ostentar la calidad de servidor público y en breves palabras "Confiere a quien por ella tiene acceso a la administración la calidad de empleado público o funcionario público, y el acto que la traduce es el nombramiento y la posesión" (Younes, 2001, p.21). La modalidad de vinculación de esta categoría de servidores ha sido denominada de esa manera porque se considera que las características que rigen tanto su vinculación como sus labores se encuentran previstas en la ley, estatutos o reglamentos -norma- y no hay posibilidad de negociarlas, al menos en principio, aunque obviamente el consentimiento debe estar presente en la relación laboral (Younes, 2001).

2 Por su finalidad, el presente estudio omite el análisis de otro tipo de servidores públicos como -funcionarios, dignatarios, servidores con contrato de prestación de servicios profesionales, servidores con contrato ocasional, entre otros-. 
En cuanto a esta modalidad la doctrina ha sido enfática en señalar que: "se manifiesta mediante la expedición de un acto administrativo de nombramiento, llámese resolución o decreto y se perfecciona con la posesión en el empleo" (Dueñas, 2009, p. 65). En el mismo sentido, los empleados públicos han sido subdivididos en dos grupos, que han sido considerados también dentro de las divisiones normativas establecidas por el legislador ecuatoriano, el primer grupo ha sido denominado como empleados de libre nombramiento y remoción. Estos empleados conforme a la doctrina "integran la órbita administrativa de la provisión discrecional, o sea aquella en la que el nominador puede escoger libremente el designado con la sola condición que este reúna los requisitos y exigencias legales" (Younes, 2001, p. 145).

En cambio, el segundo grupo son los empleados que pertenecen a la carrera administrativa o servidores de carrera, como se los conoce comúnmente, quienes ingresan a la administración a través de un proceso reglado que los hace acreedores de un nombramiento permanente. Cabe recalcar que, en la legislación ecuatoriana se ha tomado a la par un criterio objetivo que hace referencia al tipo de labores desempeñadas para esta subdivisión (Younes, 2001 y Valencia, 2015).

De esta manera ha sido determinada la forma de vinculación de estos subgrupos en la legislación ecuatoriana.

Clases de nombramientos. - Los nombramientos extendidos para el ejercicio de un puesto en la función pública pueden ser:

a) Permanentes: El que se otorga a la o el ganador del concurso de méritos y oposición, una vez que haya aprobado el período de prueba;

b) Provisionales: Aquellos otorgados para ocupar temporalmente los puestos determinados en el literal b) del artículo 17 de la LOSEP; no generarán derecho de estabilidad a la o el servidor; 
c) De libre nombramiento y remoción: Los expedidos a favor de personas que van a ocupar puestos de dirección política, estratégica o administrativa en las instituciones del Estado; y,

\section{(...)}

(Decreto Ejecutivo 710, 2011, art. 17)

Por consiguiente, los empleados de libre nombramiento y remoción son aquellos a quienes se les otorga un nombramiento ordinario. Por el contrario, los empleados de carrera son vinculados a través de un nombramiento provisional mismo que se convierte en definitivo después de haber cumplido con el periodo de prueba que se encuentre previsto. En los últimos, el vínculo se da posterior a un procedimiento administrativamente reglado que se conoce como concurso de méritos y oposición (Valencia, 2015).

Una vez tratadas las características de los empleados públicos y su correspondiente subdivisión, es necesario retomar el concepto de trabajadores oficiales. Estos servidores públicos, a pesar de no vincularse a través de ningún tipo de nombramiento anteriormente descrito, son una clase de empleados oficiales de suma importancia. La forma de vinculación de estos trabajadores es la denominada contractual, lo cual implica que se vinculan al servicio público a través de un contrato de trabajo, mismo que no es antecedido por un procedimiento reglado como en el caso del primer grupo ${ }^{3}$ sino que sus labores y condiciones se estipulan en un instrumento que se perfecciona por el consentimiento mutuo.

En conclusión, los trabajadores oficiales se vinculan por medio de un contrato laboral que "se traduce en un contrato de trabajo que regula el régimen del servicio que se va a prestar, permitiendo obviamente la posibilidad de discutir las condiciones aplicables" (Younes, 2001, p.22). Los trabajadores

3 Un sector de la doctrina se ha inclinado por la idea de que todos los servidores públicos deberían ser vinculados a través de un concurso de méritos y oposición dando cumplimiento al principio de igualdad y salvaguardando los fines de la administración pública. 
oficiales han sido mejor conocidos como obreros en la mayoría de legislaciones.

En fin, es en base a esta clasificación que antecede que los Estados deben elaborar su escalafón ${ }^{4}$ y otorgar características particulares a cada grupo según sus necesidades. Para ejemplificar de mejor manera el régimen de los empleados oficiales-servidores públicos- en la doctrina, se resumen sus características en el siguiente gráfico:

\section{Gráfico No. 1}

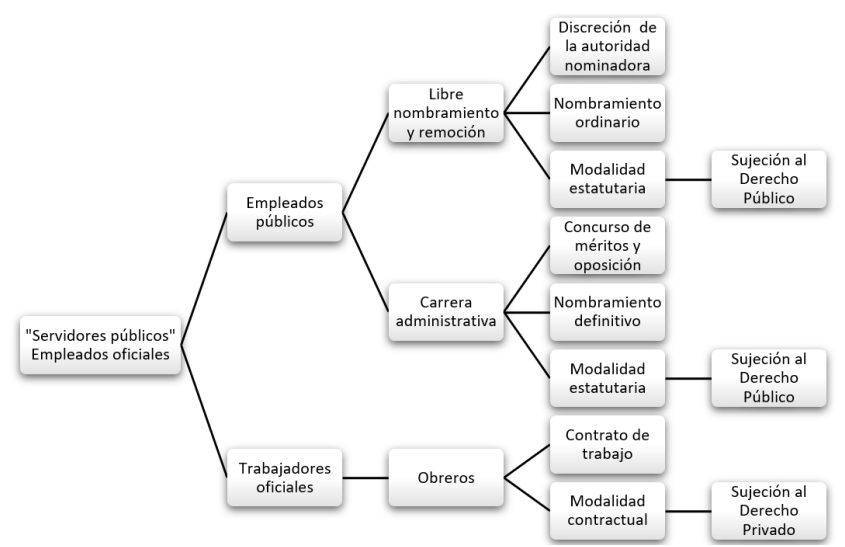

Clasificación doctrinaria y régimen laboral de los empleados oficiales (servidores públicos) (Villavicencio, 2019, p. 90)

4 Según la Resolución 081 emitida por la SENRES que trata sobre la Remuneración de Funcionarios de Nivel Superior del Sector Público -concuerda con el tipo de nombramientos establecidos por la LOSEP-, los niveles se denominarían en primer lugar, nivel jerárquico superior, el cual "está integrado por puestos con funciones y responsabilidades que involucran la toma de decisiones de carácter técnico y administrativo, cuyos titulares son los responsables de la ejecución de las políticas públicas, estatales, gubernamentales e institucionales" (Resolución 81, 2004, art.1).

El nivel medio en cambio está integrado por cargos que reúnen características determinadas por los principios que rigen a la administración pública pero además por las necesidades institucionales. El nivel técnico a su vez está integrado por aquellos servidores que pertenecen a la carrera administrativa pues poseen conocimientos técnicos para cumplir funciones específicas, lo cual en la mayoría de legislaciones les permite gozar de estabilidad en sus puestos de trabajo.

Por último, el nivel operativo es aquel conformado por aquellos cuyo trabajo es caracterizado por la preminencia de esfuerzo físico, manual o mecánico antes que el intelectual - características sumamente cuestionadas para estos trabajadores- (Valencia, 2015). 


\section{SERVIDORES PÚBLICOS EN LAS EMPRESAS PÚBLICAS}

Como ha quedado establecido, todo trabajador que preste sus servicios en una de las entidades comprendidas dentro del sector público adquiere la denominación de servidor público independientemente de la naturaleza de su vinculación. A pesar de ello, el régimen previsto para la generalidad del sector público no siempre es aplicable a los servidores, en especial cuando se trata de instituciones que por su naturaleza permiten la concurrencia del Derecho Público y el Derecho Privado en un mismo tipo de acto o contrato. Ese es el caso de las denominadas empresas públicas pues, por la finalidad que persiguen son normadas de forma particular (González, 2017).

\subsection{Aproximación conceptual a las empresas públicas}

Por la naturaleza que reviste a las empresas públicas, la doctrina ha tenido dificultad para otorgarles una concepción unánime; pese a ello, las mismas han sido dotadas de peculiaridades según la funcionalidad que han ido otorgándoles las distintas legislaciones, entre ellas la ecuatoriana. De esta manera, ya que hoy en día son consideradas como entidades de suma importancia para los planes estatales, la doctrina y la legislación han coincidido en otorgarles una definición que puede englobar todos sus elementos distintivos y pueda dar una noción de su trascendencia (Cassagne, 1998).

Las empresas industriales y comerciales del Estado como se ha denominado a las empresas públicas, "son organismos creados por la ley o autorizados por esta, que desarrollan actividades de naturaleza industrial o comercial y de gestión económica conforme a las reglas de Derecho Privado, salvo las excepciones que consagra la ley" (Villegas, 2016, p.96). En otras palabras, la doctrina ha optado por conceptualizarlas según su funcionalidad estableciendo:

La empresa industrial y comercial, como su nombre lo indica, es una empresa o expresión del Estado empresario, que incurre en actividades semejantes a las de los empresarios privados o susceptible de ser 
fundada o manejada por los empresarios particulares en la misma forma que por el Estado y por ello se regula por el derecho de los particulares, o sea el derecho privado contractual. (Villegas, 2016, p. 97)

De las definiciones que anteceden, se desprende que los elementos que hacen únicas a estas entidades devienen de la naturaleza única que poseen. Pues, si bien la actividad industrial o comercial para la cual están destinadas podría considerarse de naturaleza privada -prestación de servicios públicos o gestión de sectores estratégicos-, al ser entidades que pertenecen al sector público se genera la interrogante de si su naturaleza podría considerarse de tipo mixto. (González, 2017). Es así que la doctrina ha determinado que:

Son entidades descentralizadas que realizan actividades de índole comercial o industrial, organizadas bajo un régimen jurídico mixto, semiadministrativo y regidas alternativamente por el Derecho Público o por el Derecho Privado, según la naturaleza de sus actos. (Dromi, 2001, p.612)

Entonces, al poseer una naturaleza mixta en relación con su funcionalidad y finalidad, el régimen jurídico que la doctrina les ha otorgado también ha sido considerado como mixto. No obstante, esto ha causado gran confusión debido a que se ha considerado que debe hacerse una diferenciación en cuanto a sus actos y contratos; por ejemplo, al buscar la consecución de objetivos públicos deben someterse al Derecho Público en todo lo que refiera a su relación directa con la administración, mientras que en todo lo que atañe a las actividades de índole comercial o industrial que emprendan deben someterse al Derecho Privado (Cassagne, 1998). Finalmente se ha concluido que:

El concepto de empresa pública carece de un significado jurídico preciso; y a través de él solo puede expresarse que el Estado ha adoptado una política de intervención en el mercado, mediante distintas formas de organización que se asemejan a la empresa privada, pero ofrecen diferentes perfiles jurídicos, en función 
del grado de intervención en la economía que el Estado se propone. (Mata, 2009, p.587)

Como corolario, las empresas públicas son parte del sector público en los términos establecidos por el artículo 225 de la Constitución ecuatoriana, es decir que su creación, regulación y control depende del gobierno central y de los gobiernos autónomos descentralizados (Valencia, 2015). Obviamente, estas instituciones gozan de autonomía tal como se encuentra previsto por la legislación, razón por la cual cuentan con patrimonio propio (Cassagne, 1998). En consecuencia, las empresas públicas pertenecen al sector público de la economía ya que las rige un elemento subjetivo que es la participación directa o indirecta del Estado, y de manera objetiva su finalidad hace referencia directa a la actividad industrial o comercial para la cual estén encaminadas (Dromi, 2001).

Efectivamente, las empresas públicas pertenecen al sector público, no obstante, gozan de características que son compartidas por las empresas del sector privado lo cual, no solo las hace merecedoras de un carácter mixto, sino que también a sus actos y contratos los determina esa naturaleza particular (González, 2017). Es necesario mencionar que las empresas públicas han obtenido diversas denominaciones dentro de la doctrina $y$ la legislación comparada, pero son sus particularidades las cuales han dotado de una concepción especial a las mismas.

Por ejemplo, al analizar la ley que las rige-LOEP- en sus primeros artículos -4 y 5-, en concordancia con el texto constitucional, se ha considerado que inclinan a sus actos y contratos al sometimiento al Derecho Público, mientras que al analizar la Disposición General Primera del mismo texto normativo especial se podría considerar que tan solo pocos de sus actos y contratos -residuales- son de carácter administrativo. Lo mencionado solo da certidumbre de la naturaleza mixta caracteriza a las empresas públicas y, adicionalmente, da un breve indicio sobre la confusa regulación que las rige (González, 2017). 
Reiterando lo mencionado, el carácter mixto que caracteriza a la naturaleza de las empresas públicas es el mismo que caracteriza a sus actos y contratos. Uno de los ámbitos en los cuales ello se ha vuelto particularmente notorio y ha tomado radical importancia, ha sido el laboral. Paradójicamente, aunque las empresas públicas requieren de total interés por parte de autoridades y doctrinarios, por su naturaleza tan compleja y particular, las mismas no han sido desarrolladas ni normadas de manera adecuada. El legislador ha huido constantemente a su regulación ignorando la importancia que las reviste y las consecuencias que puede acarrear su equívoca regulación en distintas ramas del Derecho.

La legislación ecuatoriana realmente ha dejado de lado la adecuada regulación de estas entidades tratándolas de manera dispersa sin guardar concordancia con la normativa jerárquicamente superior y mucho menos con los preceptos doctrinarios; por ende, es necesario conocer brevemente sobre lo que los distintos autores han establecido respecto al régimen laboral imperante dentro de las mismas y de esta manera contrastarlo con la legislación ecuatoriana.

\subsection{Clasificación doctrinaria de los servidores públicos en las empresas públicas}

En conexión con el tema central tratado, las personas que trabajan, prestan servicios o realizan cualquier actividad inclinada a cumplir con los objetivos de las entidades que pertenecen al sector público, deben ser considerados como servidores públicos. Consecuentemente, los principios de Derecho Público como de Derecho Privado les son aplicables a estos servidores, cuando son trabajadores y ello no obsta que a sus actos y contratos también los regule un régimen jurídico especial (Cabanellas, 2008).

Como fue mencionado con antelación, para la clasificación de los servidores públicos la doctrina ha tomado en cuenta dos parámetros. El primer parámetro hace relación a la modalidad de vinculación de los servidores y la relaciona con las funciones que desarrolla cada tipo de servidor. Por el con- 
trario, el segundo criterio hace alusión al tipo de entidad al que pertenecen y prestan sus servicios. El segundo criterio es aquel que marcará la diferencia en el caso de las empresas públicas (Dueñas, 2009).

Al respecto los tratadistas se han pronunciado de la siguiente manera:

Laboralmente, quienes trabajan en las empresas industriales y comerciales del Estado, por ejercitar actividades que cualquiera puede desempeñar o semejantes a las de los particulares, regidos por el derecho de los particulares, el derecho privado o contractual, son por norma general, trabajadores oficiales. Sin embargo, los estatutos precisarán que actividades de dirección o confianza deberán ser desempeñadas por personas que tengan la calidad de empleados públicos, como excepción. (Villegas, 2013, P. 99)

Es decir que, simplemente con el fin de asimilar el régimen laboral que prima en las empresas públicas al de las empresas privadas para que las mismas puedan producir rentabilidad social y cumplir con los objetivos de su creación, por regla general los servidores públicos que laboren en las mismas deben vincularse como lo prevea el Código Laboral que sea aplicable en cada Estado. Contrario al resto del sector público, como regla general los servidores que trabajen en estas entidades serán considerados como trabajadores oficiales -modalidad contractual- dejando la forma de vinculación de los empleados públicos -modalidad estatutaria- para aquellos que de forma excepcional sean designados como servidores de libre nombramiento y remoción. Para resumir este régimen aplicable a las empresas de Estado se ejemplifica de la siguiente manera: 


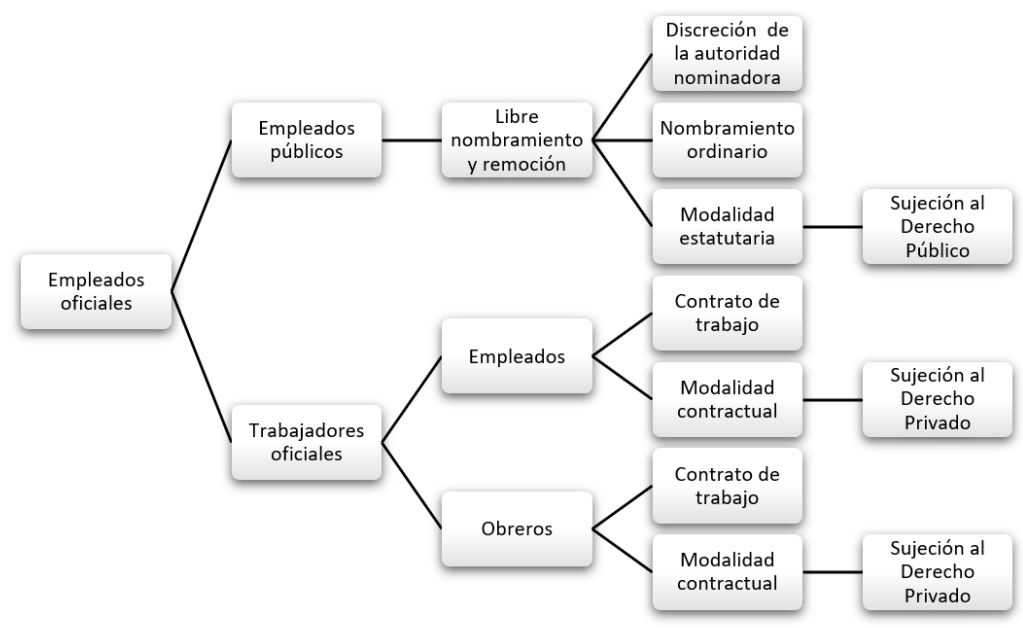

\section{Gráfico No. 2}

Clasificación doctrinaria y régimen laboral de los empleados oficiales (servidores públicos) en las empresas industriales o comerciales (empresas públicas) (Villavicencio., 2019, p. 93)

En conclusión, el régimen laboral preponderante en las empresas públicas es el de Derecho Privado, es decir que prima la modalidad contractual y los servidores son denominados trabajadores oficiales pues se vinculan a la administración a través de un contrato de trabajo y, solo excepcionalmente, los empleados públicos pueden vincularse a través de la modalidad estatutaria convirtiéndose en servidores de libre nombramiento y remoción y rigiéndose por el Derecho Público (Younes, 2001).

Este régimen resultaría simple de comprender y aplicar en la normativa respectiva, sin embargo, para el Estado ecuatoriano no ha sido sencillo. Si bien el régimen aplicable para los servidores de libre nombramiento y remoción y para los obreros coincide para todo el sector público, como está previsto en la doctrina, el régimen para los servidores de carrera debería ser tomado en consideración de forma distinta. Estos servidores deberían ser considerados como trabajadores oficiales -em- 
pleados- con el fin de que las empresas públicas no compitan en inferioridad de condiciones y que su régimen sea lo más parecido al de las empresas privadas.

\subsection{Aproximación a la regulación del talento humano en las empresas públicas ecuatorianas}

Las empresas públicas ecuatorianas gozan de una naturaleza sumamente particular, no solamente porque han sido descritas utilizando una denominación diferente a la prevista por la doctrina y por la legislación comparada, sino porque el ordenamiento jurídico ecuatoriano las ha ido dotando de elementos según sus necesidades. Estos elementos han sido escasamente concordantes, lo que implica que el legislador ha creado un régimen poco previsible y ha provocado, en ocasiones, confusión sobre la normativa aplicable. Para esclarecer las incógnitas que han nacido de esta regulación es importante analizar la norma competente para establecer su clasificación y el régimen laboral de sus servidores.

Es trascendental iniciar por conocer lo que la Constitución ecuatoriana ha plasmado acerca de estas entidades para conocer la raíz de la errónea interpretación. La Carta Magna expresa al respecto:

El Estado constituirá empresas públicas para la gestión de sectores estratégicos, la prestación de servicios públicos, el aprovechamiento sustentable de recursos naturales o de bienes públicos y el desarrollo de otras actividades económicas.

Las empresas públicas estarán bajo la regulación y el control específico de los organismos pertinentes, de acuerdo con la ley; funcionarán como sociedades de derecho público, con personalidad jurídica, autonomía financiera, económica, administrativa y de gestión, con altos parámetros de calidad y criterios empresariales, económicos, sociales y ambientales. (CRE, 2008, $\operatorname{art.315)}$ 
La Constitución deja bastante claro el objeto de creación de las empresas públicas y, al estar comprendidas en los términos del artículo 225 del mismo cuerpo legal -sector público-, es lógico que la propia administración sea aquella que regule todo lo concerniente a las mismas. En ese sentido, fue promulgada la Ley Orgánica de Empresas Públicas -LOEP- misma que hace eco de la definición antes mencionada y expresa:

Las empresas públicas son entidades que pertenecen al Estado en los términos que establece la Constitución de la República, personas jurídicas de derecho público, con patrimonio propio, dotadas de autonomía presupuestaria, financiera, económica, administrativa y de gestión. Estarán destinadas a la gestión de sectores estratégicos, la prestación de servicios públicos, el aprovechamiento sustentable de recursos naturales o de bienes públicos y en general al desarrollo de actividades económicas que corresponden al Estado. (LOEP, 2009, art.4)

En concordancia con lo mencionado en párrafos anteriores, es comprensible que los servidores que presten sus servicios o laboren en las empresas públicas sean considerados como servidores públicos pues, la propia LOEP ha hecho hincapié en lo antes expresado acerca de la naturaleza de los mismos:

NATURALEZA JURIDICA DE LA RELACION CON EL TALENTO HUMANO. - Serán servidoras o servidores públicos todas las personas que en cualquier forma o a cualquier título trabajen, presten servicios o ejerzan un cargo, función o dignidad dentro de las empresas públicas (...) (LOEP, 2009, art.18)

Consecuentemente, es necesario entender la regulación aplicable a los mismos pues, como ya quedó establecido, las empresas de Estado, empresas industriales y comerciales o empresas públicas, como las ha denominado el legislador ecuatoriano, están caracterizadas por un régimen laboral especial que les permite cumplir con los objetivos previstos. La Constitución ecuatoriana, como norma suprema ha establecido ciertos lineamientos: 


\section{(...)}

Los derechos de las servidoras y servidores públicos son irrenunciables. La ley definirá el organismo rector en materia de recursos humanos y remuneraciones para todo el sector público y regulará el ingreso, ascenso, promoción, incentivos, régimen disciplinario, estabilidad, sistema de remuneración y cesación de funciones de sus servidores.

Las obreras y obreros del sector público estarán sujetos al Código de Trabajo.

\section{(...)}

(CRE, 2008, art.229)

La Carta Magna ha establecido una guía acerca del régimen aplicable a los servidores públicos y ha reiterado que el resto de la regulación le corresponde a la normativa inferior, misma que lógicamente no puede contradecir los parámetros establecidos. La Ley Orgánica de Servicio Público fue publicada en reemplazo de la Ley Orgánica de Servicio Civil y Carrera Administrativa y, sin lugar a duda, la misma provocó un cambio significativo en la regulación del talento humano; sin embargo, a pesar de haberse promulgado de forma posterior a la Ley Orgánica de Empresas Públicas, obvió aclarar ciertos temas que lo requerían. Entre sus primeros artículos la LOSEP- ha establecido: "Las disposiciones de la presente ley son de aplicación obligatoria, en materia de recursos humanos y remuneraciones, en toda la administración pública (...)" (LOSEP, 2010, art. 3).

Lo descrito daría a entender que la LOSEP es la norma competente para regular todo lo referente al talento humano dentro del sector público, pero el mismo artículo se ha encargado de hacer una importante especificación sobre las empresas públicas, radicando la competencia para regularlas en otro cuerpo normativo: "En las empresas públicas, sus filiales, subsidiarias o unidades de negocio, se aplicará lo dispuesto en el Título IV de la Ley Orgánica de Empresas Públicas." (LOSEP, 2010, art. 3). 
La Ley Orgánica de Empresas Públicas -LOEP- ha sido designada como el cuerpo legal adecuado para normar todo lo referente a los servidores públicos que prestan sus servicios, trabajan o realizan cualquier actividad en las empresas públicas. El tema de este estudio, como ya se ha mencionado, radicará en los trabajadores que pertenecen a estas entidades, pues los demás servidores -funcionarios, dignatarios, entre otros- requieren de un análisis particular ${ }^{5}$.

La LOEP resume su competencia para el efecto, en las siguientes líneas:

Las disposiciones de la presente Ley regulan la constitución, organización, funcionamiento, fusión, escisión y liquidación de las empresas públicas que no pertenezcan al sector financiero y que actúen en el ámbito internacional, nacional, regional, provincial o local; y, establecen los mecanismos de control económico, administrativo, financiero y de gestión que se ejercerán sobre ellas, de acuerdo a lo dispuesto por la Constitución de la República. (LOEP, 2009, art.1)

Mediante un análisis somero, se entendería que la LOEP se encuentra en concordancia con la normativa y que la misma cumple con el objetivo de regular a estas entidades; sin embargo, el problema radica en el estudio a profundidad de las materias contenidas en la misma. Sin desconocer otras tantas materias reguladas de manera incompleta, la regulación al talento humano realmente ha sido deficiente pues no ha existido coherencia en el régimen impuesto y, al respecto, es necesario determinar si la LOEP cumple con su cometido.

\subsection{Clasificación y regulación de los trabajadores en las em- presas públicas ecuatorianas}

Los servidores públicos que trabajan en las empresas públicas deben dividirse en empleados públicos, que hace re-

5 Los demás servidores, entre ellos los vinculados por contratos de servicios ocasionales al igual que aquellos que poseen contratos de servicios profesionales, no serán parte de este análisis pues su relación con la administración pública requiere de un tratamiento autónomo. 
ferencia a aquellos denominados servidores de libre nombramiento y remoción y en trabajadores oficiales, que debería hacer referencia tanto a empleados -servidores públicos de carrera- y obreros. Estos parámetros deberían ser establecidos por la normativa para coincidir con la doctrina y, en especial, para que las empresas públicas puedan cumplir con su objetivo de producir rentabilidad social y no competir en inferioridad de condiciones con el resto de empresas privadas (CCE-EP-17516-SEP-CC, 2016).

En miras a regular a sus servidores públicos, la LOEP ha establecido ciertos parámetros de clasificación de los trabajadores con la finalidad de poder determinar los rasgos que los diferencian. En ese sentido, entre sus principales artículos ha establecido una clasificación que, en teoría, concuerda con el resto del bloque normativo.

\section{(...)}

La prestación de servicios del talento humano de las empresas públicas se someterá de forma exclusiva a las normas contenidas en esta Ley, a las leyes que regulan la administración pública y a la Codificación del Código del Trabajo, en aplicación de la siguiente clasificación:

a. Servidores Públicos de Libre Designación y Remoción. - Aquellos que ejerzan funciones de dirección, representación, asesoría y en general funciones de confianza;

b. Servidores Públicos de Carrera. - Personal que ejerce funciones administrativas, profesionales, de jefatura, técnicas en sus distintas especialidades y operativas, que no son de libre designación y remoción que integran los niveles estructurales de cada empresa pública; $\mathrm{y}$,

c. Obreros. - Aquellos definidos como tales por la autoridad competente, aplicando parámetros objetivos y de clasificación técnica, que incluirá dentro de este perso- 
nal a los cargos de trabajadoras y trabajadores que de manera directa formen parte de los procesos operativos, productivos y de especialización industrial de cada empresa pública. (LOEP, 2009, art.18)

El articulo precedente, además de coincidir con la clasificación otorgada por la LOSEP para los trabajadores del sector público, también concuerda con lo expresado por la doctrina. Sin embargo, el mismo hace alusión a la clasificación que ha sido establecida para la generalidad de servidores públicos, olvidando que para aquellos que prestan sus servicios en empresas públicas se requieren de un régimen especial por la naturaleza que identifica a estas entidades. Es por ello, que de este articulo parte en gran medida la confusión conceptual que ha llevado a las interpretaciones ambiguas y erróneas sobre el régimen laboral de estos servidores.

Efectivamente, las características que rigen a los servidores de libre nombramiento y remoción y a los obreros son concordantes para todo el sector público, incluidas las empresas de Estado; no obstante, los servidores de carrera gozan de un régimen distinto. En las empresas públicas, por regla general sus trabajadores deben ser considerados como trabajadores oficiales, por lo cual los servidores de carrera deberían tomar el nombre de empleados con el fin de asemejarlos funcional y gramaticalmente a lo previsto por la norma de Derecho Privado (Marienhoff, 2003).

Ya que la LOEP es la norma que en estricto sentido debería regular todos los aspectos que rigen a las empresas públicas, es necesario analizarla con mayor profundidad para entender el espíritu de la norma y concluir sobre el verdadero régimen laboral imperante. Cabe adelantar que no guarda relación con la doctrina y, en ocasiones, ni si quiera con la normativa jerárquicamente superior por lo que se requiere un análisis minucioso.

En concordancia con el criterio de clasificación según el cual la doctrina divide a los servidores públicos por su modalidad de vinculación, la LOEP ha establecido: 


\section{MODALIDADES DE DESIGNACION Y CONTRATA- CION DEL TALENTO HUMANO.}

Las modalidades de vinculación de los servidores públicos y obreros de las empresas públicas son las siguientes:

1. Nombramiento para personal de libre designación y remoción, quienes no tendrán relación laboral. Su régimen observará las normas contenidas en el capítulo II del Título III de esta Ley ${ }^{6}$ :

2. Nombramiento para servidores públicos, expedido al amparo de esta Ley y de la normativa interna de la Empresa Pública; y,

3. Contrato individual de trabajo, para los obreros, suscritos al amparo de las disposiciones y mecanismos establecidos en la Codificación del Código del Trabajo y en el contrato colectivo que se celebre.

Las normas relativas a la prestación de servicios contenidas en leyes especiales o en convenios internacionales ratificados por el Ecuador serán aplicadas en los casos específicos a las que ellas se refieren. (Ley 0, 2009, art.19)

La confusión continúa y se desarrolla a través de este artículo debido a que la norma ha establecido que, la denominación de servidores de carrera es contraria a lo establecido por la doctrina y también lo es la modalidad de vinculación. Como ya fue mencionado, el régimen de los servidores de libre nombramiento y remoción y de los obreros al ser concordante en

6 Los servidores de libre nombramiento y remoción son de suma importancia para la eficiencia y eficacia de la administración pública pues, de ellos depende en gran parte el correcto desenvolvimiento de los demás servidores, ya que cuentan con la potestad de establecer directrices y velar por la transparencia y ejecución de los objetivos del Estado. Por este motivo, se ha criticado que la LOEP establezca que los mismos no mantienen una relación laboral con la administración por el simple hecho de no pertenecer a la carrera administrativa y no gozar de estabilidad en sus puestos de trabajo (Valencia, 2015). 
todo el sector público no acarrea mayores problemas al tratarlos dentro de las empresas públicas; empero, los servidores de carrera-empleados- son aquel grupo que hace la diferencia y que determina el régimen imperante en las mismas.

La LOEP a través del mencionado artículo ha establecido que tanto a los servidores de libre nombramiento y remoción como a los servidores de carrera los rige el Derecho Público en su modalidad de vinculación a la administración pública modalidad estatutaria-. Obviando que el régimen imperante en las empresas de esta categoría es el de Derecho Privado y solo como excepción la modalidad estatutaria debe ser aplicable. ${ }^{7}$

A pesar de estas discordancias, es necesario analizar con mayor profundidad el régimen de los servidores de carrera, mismos que están regidos por el Derecho Público en su modalidad de vinculación y en su denominación. Hasta este momento, parecería que la legislación ecuatoriana confundió el régimen laboral imperante y que impuso el régimen común del sector público a las empresas públicas. Pese a ello, su articulado tampoco es concordante con esa idea pues en artículos posteriores la LOEP ha establecido que:

COMPETENCIA Y PROCEDIMIENTO. - Para efectos de la competencia y del procedimiento en las relaciones contractuales generadas entre las empresas públicas y servidores públicos de carrera y obreros, se estará a lo dispuesto en esta Ley y en el Art. 568 y siguientes de la Codificación del Código del Trabajo. (Ley 0, 2009, art.29)

$7 \quad$ El ingreso al servicio público, el ascenso y la promoción en la carrera administrativa se realizarán mediante concurso de méritos y oposición, en la forma que determine la ley, con excepción de las servidoras y servidores públicos de elección popular o de libre nombramiento y remoción. Su inobservancia provocará la destitución de la autoridad nominadora. (CRE, 2008, art.228)

En base a este artículo se ha planteado la posibilidad que todos los servidores públicos, independientemente de sus funciones y denominación, sean vinculados a través de un proceso reglado de méritos y oposición y de esa manera salvaguardar los intereses de la administración y el principio de igualdad 
Este artículo delata el régimen que ha querido imponer la normativa ecuatoriana al talento humano que presta sus servicios en las empresas públicas pues, busca que en concordancia con la doctrina tanto los servidores públicos de carrera -empleados- como los obreros, sean considerados como trabajadores oficiales y estén al amparo de la normativa de Derecho Privado, en este caso el Código del Trabajo. Es así que ha dotado de un régimen mixto a los servidores de carrera que dista del deber ser y sobre todo crea confusión pues, su modalidad de vinculación es estatutaria -reglada por la norma de Derecho Público- y la regulación de sus funciones y conflictos se da través de la norma que rige al sector privado.

Complementando la explicación que antecede, la Corte Constitucional se pronunció:

Conforme se observa, lo que ha procurado el constituyente para que efectivamente las empresas públicas no compitan en inferioridad de condiciones con el resto de empresas, es que éstas sean manejadas de diferente manera que la administración general, pues las empresas públicas buscan, entre otros objetivos, ser competitivas y con alta rentabilidad social y rendimiento, siendo una forma de gestión de recursos para lograr la consecución de los objetivos para los que fue creado el Estado, esto es, la realización de los derechos de sus coasociados.

\section{(...)}

En definitiva, el legislador, al haber dispuesto en la Ley Orgánica de Empresas Públicas, expedida por mandato de la Constitución, que sean las autoridades laborales y los jueces de trabajo los llamados a resolver las controversias que se suscitaren entre las empresas públicas y su personal (servidores públicos y trabajadores), no ha vulnerado el artículo 76 numeral 3 de la Constitución. (CC-SCN-007-11, 2011) 
Entre las diversas interpretaciones y conflictos que se han generado a raíz de lo que prevé la LOEP, se ha considerado que este cuerpo legal podría llegar a ser inconstitucional por contravenir el régimen impuesto por la LOSEP. Este criterio se ha amparado en lo que establece la Constitución en el artículo 229 que indica: "Las obreras y obreros del sector público estarán sujetos al Código de Trabajo" (CRE, 2008, art.229). Adicionalmente, se llegó a considerar que podría estar en contraposición directa con lo expresado por el artículo 326 que contiene:

16. En las instituciones del Estado y en las entidades de derecho privado en las que haya participación mayoritaria de recursos públicos, quienes cumplan actividades de representación, directivas, administrativas o profesionales, se sujetarán a las leyes que regulan la administración pública. Aquellos que no se incluyen en esta categorización estarán amparados por el Código del Trabajo. (CRE, 2008)

Por consiguiente, cabe esclarecer aquella duda con el criterio emitido por la Cote Constitucional, el cual es bastante escueto, pero para el efecto es conveniente:

(...) no existe la aparente antinomia entre la Ley Orgánica de Empresa Pública y la Ley Orgánica de Servicio Público, pues esta última, en los artículos 3 inciso final, 56 penúltimo inciso, 57 último inciso y 83 literal $\mathrm{k}$ ha reconocido el régimen propio y especial del personal de empresas públicas. (CC-SCN-007-11, 2011)

Para una mejor comprensión sobre el régimen laboral aplicable a los servidores que laboran en las empresas públicas y respecto al juez competente para resolver los conflictos que se susciten con los mismos a continuación, grafico ilustrativo: 


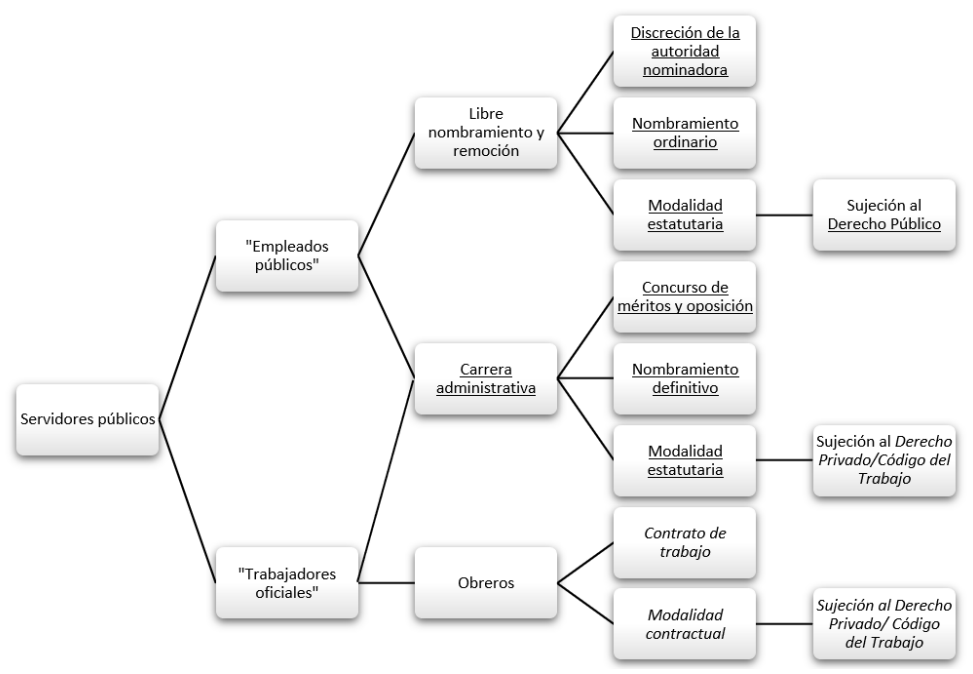

Clasificación normativa y régimen laboral de los servidores públicos que pertenecen a las empresas públicas ecuatorianas (Villavicencio, 2019, p. 98)

Una vez aclarado el hecho que el régimen laboral impuesto en las empresas públicas, a través de la LOEP, ha sido aceptado por la Corte Constitucional, es menester resumir lo que ha previsto el ordenamiento jurídico ecuatoriano -en contradicción con la doctrina-. Si bien se ha establecido que los servidores de libre nombramiento y remoción se vinculan a través de una modalidad estatutaria -nombramiento- y los rige la norma de Derecho Público -LOSEP y LOEP- mientras que los obreros se vinculan a través de una modalidad contractual -contrato de trabajo- y los rige la norma de Derecho Privado -Código del Trabajo-; se ha determinado que a los servidores de carrera los ampara un régimen laboral mixto. Mediante este régimen se establece que a su modalidad de vinculación -estatutaria- y a su denominación los regula la norma de Derecho Público, mientras que la regulación de sus funciones se da en concordancia con el Derecho Privado. 
Con la estricta finalidad de corroborar que el régimen imperante en las empresas públicas ha sido el de Derecho Privado y que lo previsto por la LOEP ha sido producto de una confusión conceptual, es necesario traer a colación el anterior texto constitucional. A pesar de que en aquel tiempo las empresas públicas no tenían la connotación que hoy en día poseen, la Carta Constitucional vigente en 1979 ya establecía un régimen laboral concordante con la doctrina y al respecto estableció:

Art. 125.- Para la elaboración y ejecución de los planes de desarrollo del Estado se considerarán como entidades del sector público, las siguientes:

a) los diferentes organismos y dependencias administrativas del Estado;

b) las entidades, que integran la administración provincial o cantonal, dentro del régimen seccional;

c) las personas jurídicas creada por ley para el ejercicio de la potestad estatal o para la prestación de servicios públicos o, para actividades económicas asumidas por el Estado y las creadas por acto legislativo seccional para la prestación de servicios públicos.

\section{(...)}

Las relaciones entre los organismos comprendidos en las letras a) y b) o de instituciones creadas por ley para el ejercicio de la potestad estatal y sus servidores, se sujetan a las leyes que regulan la administración Pública, salvo a las que se refieren al sector laboral determinadas en el Código del Trabajo. Las personas jurídicas creadas por ley o por acto legislativo seccional para la prestación de servicios públicos o las creadas para actividades económicas asumidas por el Estado, norman las relaciones con sus servidores de acuerdo con el Código del Trabajo, a excepción de las personas que ejerzan funciones de dirección, gerencia, representación, asesoría, jefatura departamental o similares, las cuales están sujetas a las leyes que regulan la administración pública. (CPE, 1979) 
Para concluir con el análisis de la normativa aplicable a la regulación del talento humano en las empresas públicas es menester aclarar lo que ha expresado la LOEP en su artículo 17 , mismo que ratifica la competencia de la misma para normar todo lo concerniente a los servidores públicos y también recalca los cuerpos normativos de aplicación concurrente que son la LOSEP y el Código del Trabajo.

NOMBRAMIENTO, CONTRATACION Y OPTIMIZACION DEL TALENTO HUMANO. - La designación y contratación de personal de las empresas públicas se realizará a través de procesos de selección que atiendan los requerimientos empresariales de cada cargo y conforme a los principios y políticas establecidas en esta Ley, la Codificación del Código del Trabajo y las leyes que regulan la administración pública. Para los casos de directivos, asesores y demás personal de libre designación, se aplicarán las resoluciones del Directorio.

El Directorio, en aplicación de lo dispuesto por esta Ley, expedirá las normas internas de administración del talento humano, en las que se regularán los mecanismos de ingreso, ascenso, promoción, régimen disciplinario, vacaciones y remuneraciones para el talento humano de las empresas públicas. (LOEP, 2009, art.17)

La LOEP hace referencia a las diferentes normas analizadas con antelación, pero en esta ocasión también incluye a las resoluciones del Directorio y, al respecto, es importante aclarar una interrogante que ha causado mayor confusión y que, a pesar de ya haber sido absuelta, sigue generando aplicaciones contrapuestas. En varios criterios se ha considerado que el Directorio puede imponer el régimen que rija dentro de las empresas públicas sin tomar en consideración lo que dicta la normativa ya establecida, lo cual por simple deducción resulta ilegal e ilegitimo y en se sentido se ha pronunciado la Procuraduría General del Estado: 
1.- La gestión del talento humano de la empresa pública se rige por la normativa que para atender los requerimientos empresariales específicos expida el Directorio de la entidad, en aplicación del inciso segundo del artículo 17 de la Ley Orgánica de Empresas Públicas, normativa que debe guardar conformidad con esa Ley, el Código del Trabajo y las demás leyes que rigen para toda la administración pública y que en consecuencia, está sujeta al control posterior del Ministerio de Relaciones Laborales conforme al inciso final del citado artículo 17 de la Ley Orgánica de Empresas Públicas. (OF. PGE. 11163; 2012)

En conclusión, es necesario entender que el estatuto o reglamento interno de cada empresa expedido por el Directorio de la misma, tiene la potestad de desarrollar la normativa vigente pero no pude contradecirla. Ha existido confusión respecto del estatuto orgánico, como es conocido en la doctrina la ley especial que rige a las entidades del sector público y que en este caso se trata de la LOSEP o la LOEP, según la entidad regulada. A estos cuerpos legales les correspondería determinar "exceptivamente cuales actividades de dirección y confianza en las empresas industriales comerciales del Estado deben ser desarrolladas por personas que tenga la calidad de empleados público, pues legalmente, por norma general son trabajadores oficiales" (Younes, 2001, p. 99).

En conclusión, la LOEP, como estatuto orgánico, debería regular todo lo referente a los servidores que laboran en las empresas públicas ecuatorianas. Sin embargo, la misma cuenta con un número escaso de artículos dedicados a la regulación del talento humano, entre los cuales ha establecido un régimen poco adecuado. La LOEP se ha limitado a designar a varios cuerpos legales la potestad de normar a los trabajadores según la clasificación mixta que les ha otorgado. En otras palabras, en primer lugar, ha dotado de un régimen discordante a sus servidores y, en segundo lugar, no los ha normado a cabalidad. Obviamente esa deficiencia no puede ser solventada por el Directorio, mismo que no puede suplir esa deficiencia de la LOEP y solo puede limitarse a expedir normativa que desarrolle lo establecido por la LOSEP, LOEP y el Código del Trabajo. 
Concretamente la falta de claridad en la normativa ha acarreado no solo interpretaciones arbitrarias entre los administradores de justicia y autoridades administrativas, sino que ha incurrido en violaciones concomitantes de derechos constitucionales. Un ejemplo claro ha sido el ejercicio de la acción de despido ineficaz, misma que ha sido prevista por el Código del Trabajo para efectivizar la garantía de despido ineficaz. Los trabajadores amparados por este cuerpo normativo han podido ejercer esta acción a través de un procedimiento sumarísimo y reclamar sus derechos vulnerados.

Mientras que, por el contrario, al encontrarse prevista por la LOSEP la generalidad de servidores de carrera y de libre nombramiento y remoción no han podido ejercerla directamente y han tenido que reclamar sus derechos a través de una acción constitucional o ejercer una acción ante las autoridades contencioso administrativas. Debido a ello, la situación concreta de los servidores de carrera que prestan sus servicios en las empresas publicas ecuatorianas se ha caracterizado por la incertidumbre pues, coexisten sentencias de jueces laborales que han establecido ser competentes para conocer las acciones de despido ineficaz que han ejercido estos servidores mientras que otros han establecido que la competencia les pertenece a los tribunales contencioso administrativos y que dicha acción no puede ser ejercida por este tipo de trabajadores debido a la entidad a la que pertenecen.

En fin, esta problemática ha tenido que ser absuelta vagamente por la jurisprudencia y la doctrina. Sin embargo, una solución real implica que la LOEP sea el cuerpo normativo que contenga todos los lineamientos sobre los servidores públicos que laboren en las empresas públicas, regulando a los servidores de carrera y a los obreros según lo que ha previsto el Código del Trabajo y a los servidores de libre nombramiento y remoción según lo previsto en la LOSEP.

Posiblemente, el legislador aspiró a la expedición de un reglamento a la Ley Orgánica de Empresas Públicas que regule estos aspectos. No obstante, 10 años después dicho anhelo no se ha visto plasmado y por ello, es necesario tener en claro 
la regulación aplicable a través del análisis de la normativa vigente y relacionarla con los preceptos doctrinarios que ayudan a esclarecer, en este caso, el deber ser de la normativa. Es de suma importancia determinar con claridad el régimen aplicable a cada tipo de servidor y como tal el cuerpo legal que los ampara, no solo por seguridad jurídica sino por garantizar el debido proceso y la tutela judicial efectiva.

\section{CONCLUSIONES}

Este artículo ha cumplido con el objetivo de esclarecer el régimen laboral aplicable a los trabajadores que prestan sus servicios en las empresas públicas ecuatorianas. Al respecto se han generado las siguientes conclusiones:

La Constitución ecuatoriana ha establecido expresamente que los obreros que pertenezcan al sector público estarán regidos por el Código del Trabajo; no obstante, ha establecido que la norma infra constitucional debe regular al talento humano en el resto de su clasificación y características. La LOSEP ha hecho referencia expresa a la competencia de la LOEP para regular a los servidores públicos que pertenecen a las empresas públicas ecuatorianas; en ese sentido, la LOEP a la vez ha indicado que existen cuerpos legales de aplicación concurrente en la regulación del talento humano, entre ellos, la LOSEP, el Código del Trabajo y las resoluciones emitidas por el Directorio de cada empresa, los cuales no deben contradecirse. Concordancias: LOEP art.29; CRE. arts. 229 y 326 y LOSEP arts. 3, 56, 57 y 83.

Los servidores públicos que trabajan en las empresas públicas ecuatorianas poseen un régimen laboral distinto al previsto para el resto del sector público. Si bien el régimen aplicable a los servidores de libre nombramiento y remoción, vinculados a través de una modalidad estatutaria y regidos por el Derecho Público y el régimen de los obreros, vinculados a través de una modalidad contractual y regidos por el Derecho Privado, concuerda; el resto de servidores deben estar regulados por norma general por el Derecho Privado para cumplir con los objetivos del Estado como empresario. Concordancias: CRE art. 225 y 315; LOEP arts. 4 y 5 . 
La legislación ecuatoriana ha otorgado a las empresas públicas un régimen laboral que dista del recomendado por la doctrina pues, ha determinado que los servidores de carrera sean vinculados a través de una modalidad estatutaria - concurso de méritos y oposición / nombramiento- que está regulada por el Derecho Público, mientras que sus relaciones con la administración sean reguladas por el Derecho Privado -Código del Trabajo-. Consecuentemente, se ha establecido un régimen laboral mixto en el que confluyen el régimen de Derecho Privado y Público en una misma clase de servidores, regulación que ha credo confusión, pese a que dicho régimen ya ha sido declarado como constitucional. Concordancias: LOEP arts. 17, 19 y 29

Debido a que el Derecho Privado impera en las relaciones de los servidores de carrera y obreros con las empresas públicas, el juez del trabajo es el competente para resolver los conflictos que devengan de dicha relación laboral, mientras que el juez contencioso administrativo -residual- es competente para resolver los conflictos que se presenten con los servidores de libre nombramiento y remoción. Dicha competencia ha sido ratificada por la Corte Constitucional. Concordancias: LOEP art. 32 y CRE art. 76.

\section{REFERENCIAS BIBLIOGRÁFICAS}

Bielsa, R. (1964). Derecho Administrativo. Buenos Aires: La Ley.

Cabanellas, G. (2008). Diccionario enciclopédico de derecho usual (Vol. VIII). Buenos Aires, Argentina: Heliasta.

Cassagne, J. (1998). Derecho administrativo (Vol. I). Buenos Aires, Argentina: Abeledo Perrot.

Dromi, R. (2001). Derecho Administrativo (Novena ed.). Buenos Aires: Editorial de Ciencia y Cultura.

Dueñas Quevedo, C. C. (2009). Derecho Administrativo Laboral. Bogotà: Ibañez. 
Gonzalèz, F. (2017). Las Empresas Públicas en el Ecuador: Su situación jurídica y su régimen laboral. Cuenca: (Tesis de grado). Universidad de Cuenca. Recuperado de http://dspace.ucuenca.edu.ec/bitstream/123456789/27275/1/ Libro\%20Empresas\%20Públicas.pdf

Krotoschin, E. (1977). Tratado práctico del derecho del trabajo (Vol. I). Buenos Aires, Argentina: Desalma.

Marienhoff, M. (2003). Tratado de Derecho Administrativo (Cuarta ed.). Buenos Aires: Editorial Abeledo-Perrot.

Mata, I. (2009). Ensayos de Derecho Administrativo. Buenos Aires: Ediciones RAP.

Sector público, con nuevas reglas para contratación de trabajadores. (08 de diciembre de 2015). El Universo. Recuperado de https://www.eluniverso.com/noticias/2015/12/08/nota/5286669/sector-publico-nuevas-reglas-contratacion-trabajadores

Trujillo, J. C. (2008). Derecho del Trabajo (Vol. I). Quito, Ecuador: Centro de Publicaciones PUCE.

Valencia, L. (2015). La problemática del derecho a la estabilidad y permanencia de los servidores. Quito: (Tesis de maestrìa). UASB.

Villegas Arbelàez, J. (2013). Derecho Administrativo Laboral: Principios, estructura y relaciones individuales. Bogotà: Legis.

Villavicencio, L. (2019). Fundamento y Exigibilidad de la Garantía de Despido Ineficaz de las Trabajadoras en Estado de Gestación y Período de Lactancia en las Empresas Públicas Ecuatorianas (Tesis de pregrado). PUCE, Quito-Ecuador 
Younes Moreno, D. (2001). Derecho Administrativo Laboral (Novena ed.). Bogotà: TEMIS.

\section{REFERENCIAS NORMATIVAS (LEYES, DECRETOS, RESO- LUCIONES, SENTENCIAS)}

Asamblea Nacional del Ecuador. Ley Orgánica de Empresas Publicas. [Ley 0 de 2009]. (16 de octubre de 2009). RO. 48 de 16 de octubre de 2009.

Asamblea Nacional del Ecuador. Ley Orgánica de Servicio Público. [Ley 0 de 2010]. (06 de octubre de 2010). RO. 294 de 06 de octubre de 2010.

Congreso Nacional del Ecuador. Código del Trabajo. [Codificación 17 de 2005]. (16 de diciembre de 2005). RO. 167 de 16 de diciembre de 2005.

Constitución de la República del Ecuador [Const.]. (2008). [Reformada] Recuperado de http://www.mdconsult. internacional.edu.ec:2052/Webtools/LexisFinder/ DocumentVisualizer/FullDocumentVisualizerPDF.aspx?id=PUBLICO-CONSTITUCION_DE_LA_REPUBLICA_DEL_ECUADOR

Constitución Política del Ecuador [Const.]. (1979). [Derogada] Recuperado de http://www.mdconsult.internacional. edu.ec:2052/Webtools/LexisFinder/DocumentVisualizer/FullDocumentVisualizerPDF.aspx?id=CONSTITU-CONSTITUCION_POLITICA_DEL_ANO_1979

Corte Constitucional del Ecuador. (01 de agosto de 2018) Sentencia N. ${ }^{\circ}$ 018-18-SIN-CC [MP Alfredo Ruíz].

Corte Constitucional del Ecuador. (19 de octubre de 2016) Sentencia N. ${ }^{\circ}$ 175-16-SEP-CC [MP Ruth Seni].

Corte Constitucional del Ecuador. (31 de mayo de 2011) Sentencia N. ${ }^{\circ}$ 007-11-SCN-CC [MP Patricio Herrera]. 
Presidencia de la República del Ecuador. Reglamento General a la Ley Orgánica del Servicio Público. [Decreto 710 de 2011]. (01 de abril de 2011). RO. 418 de 01 de abril de 2011.

Procuraduría General del Estado. Extracto de consulta Empresa Pública Municipal de Aseo de Machala, EMAM EP. [Oficio 11163 de 2012]. (20 de diciembre de 2012).

SENRES. Remuneración de Funcionarios de Nivel Superior del Sector Público. [Resolución 81 de 2004]. (09 de julio de 2004). RO. 374 de 09 de julio de 2004.

Recibido: $16 / 04 / 2020$

Aprobado: 12/06/2020

Lizzeth Karina Villavicencio Logroño: Investigadora jurídica independiente

Correo electrónico: lizzethvillavicenciol@gmail.com

Ciudad: Quito

País: Ecuador 
